\title{
Potential Adiponectin Receptor Response Modifier Therapeutics
}

\author{
Laszlo Otvos Jr. ${ }^{1,2,3 *}$ \\ ${ }^{1}$ OLPE LLC, Audubon, PA, United States, ${ }^{2}$ Allysta Pharmaceuticals, San Mateo, CA, United States, ${ }^{3}$ Institute of Medical \\ Microbiology, Semmelweis University, Budapest, Hungary
}

\section{OPEN ACCESS}

Edited by:

Tania Fiaschi,

University of Florence, Italy

Reviewed by:

Gary Sweeney,

York University, Canada

Carsten Grötzinger,

Charité Medical University of

Berlin, Germany

*Correspondence:

Laszlo Otvos Jr.

lotvos@comcast.net

Specialty section:

This article was submitted to

Translational Endocrinology,

a section of the journal

Frontiers in Endocrinology

Received: 10 May 2019

Accepted: 19 July 2019

Published: 13 August 2019

Citation:

Otvos L Jr (2019) Potential Adiponectin Receptor Response

Modifier Therapeutics.

Front. Endocrinol. 10:539. doi: 10.3389/fendo.2019.00539
Many human diseases may benefit from adiponectin replacement therapy, but due to pharmacological disadvantages of the intact protein, druggable options focus on peptidic, and small molecule agonists of the adiponectin receptor. Peptide-based adiponectin replacement drug leads are derived from, or resemble, the active site of globular adiponectin. ADP355, the first-in-class such peptide, exhibits low nanomolar cellular activities, and clinically acceptable efficacies in a series of fibrotic and inflammation-derived diseases. The advantage of small molecule therapies, spearheaded by AdipoRon, is oral availability and extension of utility to a series of metabolic conditions. It is exactly the difficulties in the reliability and readout of the in vitro measures and the wealth of in vivo models that make comparison of the various drug classes complicated, if not impossible. While only a fewer number of maladies could take advantage of adiponectin receptor antagonists, the limited number of these available can be very useful tools in target validation studies. Alternative approaches to direct adiponectin signaling control use upstream adiponectin production inducing therapies but currently these offer relatively limited success compared to direct receptor agonists.

Keywords: active site, agonist, antagonist, fibrosis, insulin sensitivity, nanomolar activity, oral efficacy, signaling

\section{THE NEED FOR PEPTIDE- AND SMALL MOLECULE-BASED ADIPONECTIN RECEPTOR RESPONSE MODIFIERS}

Adiponectin, a 244 amino acid cytokine, normally produced by the fat tissue is known to exert beneficial effects in a series of human conditions, including insulin resistance, cardiovascular disease, inflammatory conditions and cancer (1-3). The human adiponectin protein is comprised of an $\mathrm{N}$-terminal signal sequence, a variable region, a collagenous domain, and a C-terminal globular domain [(4); Figure 1]. While monomeric forms are infrequently observed, adiponectin protein mainly circulates in trimeric, hexameric and higher order complexes (5). The two adiponectin receptors that are responsible for downstream signaling, AdipoR1 and AdipoR2, are seven-transmembrane domain proteins containing an intracellular domain at the $\mathrm{N}$-terminus and a C-terminal extracellular domain $(6,7)$.

Due to the generally beneficial effects of the adipokine, a series of human conditions could use adiponectin replacement therapy (8). Prior investigations of adiponectin suggest multiple targets for clinical intervention. Decreased circulating adiponectin concentrations can increase the risk developing type 2 diabetes, metabolic syndrome, atherosclerosis, and cardiovascular disease in obese patients and need to be restored to normal levels $(9,10)$. Hypoadiponectinemia has also been associated with increased risks of several cancers and 


\section{$\mathrm{N}$-terminus}

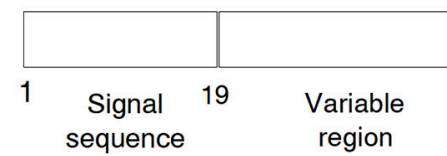

C-terminus

149 Identified 166 active site
244

$\begin{array}{lll}\text { Collagenous } & 108 \quad \text { Globular domain }\end{array}$

domain

FIGURE 1 | Domain structure of monomeric human adiponectin protein. While multimerization takes place at the N-terminal regions, the identified active site is located at the C-terminal globular fragment.

correlated with poor long-term prognosis (11). Circulating adiponectin levels are inversely related to inflammation indicating potential therapeutic utility in various conditions characterized with inflammatory processes (12). Adiponectin has demonstratable an anti-fibrotic action in the liver and it was suggested that adiponectin may be developed as a new therapeutic candidate for the treatment of liver fibrosis (13). Lastly, adiponectin impacts important brain functions including energy homeostasis, hippocampal neurogenesis and synaptic plasticity (14). Overall, multiple studies indicate indicate the potential value of adiponectin replacement therapy in the pathohysiology of neuroprotective, antiatherogenic, and antidepressant diseases.

However, there are two salient challenges in converting adiponectin protein or slightly modified versions into a viable drug candidate. Firstly, the heterogeneity of the expressed protein structures (15) prevent highly reproducible results in vitro and in vivo (16). Secondly, the extreme insolubility of the C-terminal domain and larger peptide fragments thereof (17) prevent druggable protein-based lead generation. Full-length adiponectins produced by mammalian cells, as opposed to bacterially expressed versions, are fully active insulin sensitizers due to post-translational hydroxylation and subsequent glycosylation of lysine residues at the central collagenous domain (18). Adiponectin-based therapeutic modulators are consequently currently unavailable. Luckily, peptide- and small molecule-based adiponectin receptor response modifiers can fill the denoted gap toward clinical development of adiponectin replacement therapies.

\footnotetext{
Abbreviations: ACC, acetyl-CoA carboxylase; AdipoR, adiponectin receptor; AdipoRon, orally available small molecule adiponectin receptor agonist; ADP355, first generation adiponectin receptor peptide agonist; ADP399, second generation peptidic adiponectin receptor agonist, linear dimer of ADP355; ADP400, adiponectin receptor antagonist/inverse agonist; AKT, protein kinase B; AMPK, $5^{\prime}$ adenosine monophosphate-activated protein kinase; C/EBP, CCAAT enhancer binding protein; CCK, cholecystokinin; EC, effective concentration; eNOS, endothelial nitric oxide synthase; ERK, extracellular-signal-regulated kinase; FAK, focal adhesion kinase; gAd, adiponectin protein C-terminal globular domain; GTDF, designer quercetin analog; HSC, hepatic stellate cells; IC, inhibitory concentration; IL, interleukin; $\mathrm{K}_{\mathrm{d}}$, dissociation constant; LDLR, low-density lipoprotein receptor; MAPP, ceramidase inhibitor; PCSK9, proprotein convertase subtilisin/kexin type 9; PPAR, peroxisome proliferator-activated receptor; siRNA, small interfering ribonucleic acid; SMA, $\alpha$-smooth muscle actin; SMAD, signal transducers for receptors of the transforming growth factor $\beta$; STAT3, signal transducer and activator of transcription 3; TGF, transforming growth factor; TIMP, tissue inhibitor of metalloproteinases; TNF, tumor necrosis factor; VSMC, vascular smooth muscle cells.
}

\section{DIFFICULTIES IN SCREENING}

Comparison of effectors in adiponectin replacement therapies further complicated is by (a) a litany of adopted screening assays (i.e., specific in vitro efficacies, various signaling effects, downstream activities) various research groups employ in the enormous number of related reports (19), and (b) the inconsistencies in any given assay looking for downstream effects. For example, cell-type dependence of AMPK activity (perhaps the most frequently used assay for testing adiponectin receptor activation) during adiponectin signaling is reported together with differences in the cellular effects of various adiponectin protein preparations (20). In general, the truthful evaluation of the assay readouts is not without controversy. Many tissues produce adiponectin and locally produced adiponectin can interfere with autocrine or paracrine activities that will result in either false positive or false negative screening assays (21). The proposed alterations in signaling due to differing activation of the two slightly different receptor types (22) interferes with the evaluation of potential adiponectin receptor response modifiers. Age-dependent alterations in adiponectin activity (23) and the involvement of accessory molecules (24) add yet additional aspects to screening and diagnostic difficulties.

Agents with multiple effects also inducing endogenous adiponectin production can exert effects opposite to those predicted, as was shown on acetaldehyde during fibrogenesis in hepatic stellate cells (25). Moreover, adiponectin functions in mouse primary hepatocytes appear to be independent of signaling events observed in vivo (26). Furthermore, the extent of adiponectin-induced smooth muscle cell proliferation depends upon proteolytic processing into shorter forms (27).

This review intends to critically evaluate the various adiponectin-replacement therapy options. To make the results more comparable, when $\mathrm{EC}_{50}$ or $\mathrm{IC}_{50}$ figures were not directly provided in the publications, this author calculated them from the available figures or tables. However, it is beyond the scope of this review to validate the in vitro assays and the actual data. The interpretation of the results in the individual reports are taken here prima facie. Even so, once preclinical development is underway unforeseen opportunities may arise. The journey of peptidomimetic ADP355 (vide supra) from a potential systemic cancer therapeutic to impending clinical trials as eye drops against dry eye disease (28) provides confidence that systematic research on adiponectin functions and potential adiponectin receptor response modifiers may lead to novel therapeutics. 


\section{THE ACTIVE SITE OF ADIPONECTIN AND FIRST-IN-CLASS ADIPOR AGONIST PEPTIDOMIMETICS}

The first step for designing peptidic agonists of adiponectin receptors was the identification of the active site within the native protein (29). Multiple accounts suggest that the C-terminal globular domain (gAd) carries the active site because this domain exhibits powerful metabolic effects in various tissues $(30,31)$. Overlapping 10-mers identify an extended stretch of amino acid residues (149-166) that inhibit cancer cell proliferation with the central Ile-Pro-Gly-Leu-Tyr-Tyr-Phe-Ala octapeptide representing the minimally active site [(29); Table $\mathbf{1}]$.

A targeted peptide-type library containing non-natural residues to improve metabolic stability and solubility yielded peptidomimetic ADP355, H-DAsn-Ile-Pro-Nva-Leu-Tyr-DSerPhe-Ala-DSer- $\mathrm{NH}_{2}$ (Table 1), that at $100 \mathrm{nM}$ concentration inhibits cancer cell growth better than gAd at $50 \mathrm{ng} / \mathrm{mL}$ $(\sim 3.5 \mathrm{nM})$. In agreement with most full-sized adiponectinrelated studies, ADP355 increases AMPK and STAT3 phosphorylation in MCF-7 breast cancer cells and inhibits ERK1/2 phosphorylation in MDA-MB-231 triple negative breast cancer cells. A 13-mer peptide derived from the collagen domain of adiponectin also activates AMPK and improves glucose and fatty acid metabolisms but with an $\mathrm{EC}_{50}$ of $4 \mathrm{mM}$ on enhancing glucose uptake (32) it cannot be considered a true AdipoR activator (please note the discrepancy between $\mathrm{X}$-axis labeling in $\mu \mathrm{g} / \mathrm{mL}$ and actual data in $\mathrm{mg} / \mathrm{mL}$ ).

ADP355 exhibits adiponectin-like activities in several in vitro and in vivo assays. The peptide inhibits renal fibroblast differentiation with an $\mathrm{IC}_{50}$ of $\sim 50 \mathrm{nM}$ concentration (33). In agreement with the reversal of renal fibrosis, ADP355 inhibits fibroblast migration and attenuates fibrotic responses in threedimensional human skin models together with attenuating constitutive fibrotic gene expression in unstimulated systemic sclerosis fibroblasts (34). Both adiponectin protein and ADP355 suppress phosphorylation of focal adhesion kinase (FAK) in

TABLE 1 | Sequences of peptidic adiponectin receptor response modifiers.

\begin{tabular}{ll}
\hline Peptide & Sequence \\
\hline AGONISTS & \\
$\begin{array}{l}\text { Globular adiponectin } \\
\text { active site }\end{array}$ & His-Cys-Asn-Ile-Pro-Gly-Leu-Tyr-Tyr-Phe-Ala-Tyr-His-lle \\
ADP355 & Asn-Ile-Pro-Nva-Leu-Tyr-Ser-Phe-Ala-Ser \\
ADP399 & $\begin{array}{l}\text { Asn-lle-Pro-Nva-Leu-Tyr-Ser-Phe-Ala-Ser-His-Pro-Dab- } \\
\text { Asn-lle-Pro-Nva-Leu-Tyr-Ser-Phe-Ala-Ser-His-Pro }\end{array}$ \\
Pep70 & $\begin{array}{l}\text { Pro-Gly-Leu-Tyr-Tyr-Phe-Asp } \\
\text { BHD1028 }\end{array}$ \\
Tyr-Tyr-Phe-Ala-Tyr-His-Pro-Asn-lle-Pro-Gly- \\
ANTAGONIST & Leu-Tyr-Tyr-Phe \\
ADP400 & Chex-Gly-Leu-Tyr-Ser-Phe-Ala-Ser \\
\hline
\end{tabular}

Dab is 2,4-diamino butyric acid, L-Nva is norvaline, Chex is 1-amino-cyclohexyl carboxylic acid. D-amino acids are printed in italics. All designer peptides have free-amino termini and are C-terminal amides. hepatic stellate cells (HSC) assessed by immunofluorescent imaging and quantitation. Because HSCs are precursors for hepatic fibrosis, ADP355 might be an effective therapy option for liver fibrosis (35). In a follow-up study, ADP355 was conjugated to gold nanoparticles and injected intraperitoneally at $1 \mathrm{mg} / \mathrm{kg}$ dose (36). Liver fibrosis markers, including serum aspartate aminotransferase, alanine aminotransferase and hydroxyproline are attenuated, suggesting that ADP355 is a potent anti-fibrotic agent and potentially an effective intervention against liver fibrosis. In support, while chronic $\mathrm{CCl}_{4}$-treatment induces liver fibrosis, ADP355 treatment significantly reverses this process. Key markers for fibrogenesis ( $\alpha$-smooth muscle actin, $\alpha$ SMA, transforming growth factor- $\beta 1$, TGF- $\beta 1$, connective tissue growth factor, and the tissue inhibitor of metalloproteinase I, TIMP1) are markedly attenuated (36). ADP355 also activates proprotein convertase subtilisin kexin type 9 (PCSK9) and peroxisome proliferator-activated receptor $\gamma(\operatorname{PPAR} \gamma)$ expression at $50 \mathrm{nM}(37)$.

When administered intraperitoneally at $1 \mathrm{mg} / \mathrm{kg} /$ day for 14 days to mice with protease inhibitor-induced lipodystrophy, peptide ADP355 restores the subcutaneous adipose tissue and reverses hyperinsulinemia, hypertriglyceridemia, and hypoadiponectinemia (38). In addition, ADP355 prevents protease inhibitor-induced cognitive impairment and brain injury in mice. ADP355 activates hepatic LDLR expression and ameliorates lipid metabolism in both wild type and apoE ${ }^{-/-}$mice and inhibits atherosclerosis in $\mathrm{apoE}^{-/-}$mice at 1 $\mathrm{mg} / \mathrm{kg} /$ day administered for 12 weeks (37). Upon intraperitoneal injection into scid mice xenotransplanted with MCF-7 breast cancer tumor, ADP355 therapy at a $1 \mathrm{mg} / \mathrm{kg} /$ day dose decreases tumor size by $31 \%$ compared to untreated animals (29).

In a bleomycin-induced mouse skin fibrosis model, ADP355 treatment mitigates the increase in dermal thickness, collagen accumulation, fibrotic gene expression, and dermal white adipose tissue attrition at both 0.2 and $1 \mathrm{mg} / \mathrm{kg} /$ day intraperitoneal doses (34). Liver lysates of mice with liver fibrosis and treated with ADP355 display typical adiponectin-mediated signaling changes such as increased phosphorylation of both endothelial nitric oxide synthase (eNOS) and AMPK as well as decreased phosphorylation of AKT (36). The ADP355 dose in this instance was $0.5 \mathrm{mg} / \mathrm{kg}$ intraperitoneally every other day for 14 days. Finally, ADP355 mimics adiponectin action in thioacetamide-induced liver injury (39). ADP355 treatment increases liver glycogen, decreases serum alanine transaminase and alkaline phosphatase activity, and promotes body weight gain, hyper-proliferation and hypo-apoptosis. In addition, $1 \mathrm{mg} / \mathrm{kg}$ intraperitoneal ADP355 administration suppresses activation of HSC and liver macrophages. The signaling events are inactivation of TGF- $\beta 1 /$ SMAD2 and the promotion of AMPK and STAT3 phosphorylation.

Further optimization attempts of ADP355 failed to yield derivatives with notably improved cellular activities $(33,40)$. Neither chain extension to the termini, nor reinstallation of native residues, even cyclization of the potent dimer, lead to enhanced activity profile. Incorporation of Pro and Hyp residues changes the conformation to a structure that increasingly resembles to the active native $\beta$-sheet. However, the 10 -fold 
improved activity against cancer cell proliferation did not carry over to any enhanced activity in the ophthalmic applications in vivo. Switching the ADP355 drug lead that possesses remarkable low dose in vivo efficacy and excellent toxicity profiles in various animal models is therefore not warranted.

\section{ADDITIONAL PEPTIDIC ADIPOR AGONISTS}

The C-terminal decapeptide fragment of the active site, termed peptide 27, exhibits in vitro and in vivo advantages very similar to those elicited by ADP355 in the skin fibrosis model (34). Peptide 27 treatment is associated with increased AMPK phosphorylation in skeletal muscle. Based on the crystal structure of AdipoR1, peptide agonists were designed using proteinpeptide docking simulations (41). The sequence of the best hit, BHD-1028 is remarkably similar to a dimeric peptide 27 segment (Table 1). BHD-1028 induces AMPK phosphorylation in differentiated $\mathrm{C} 2 \mathrm{C} 12$ myotubes at as low as $800 \mathrm{nM}$ concentration. Dimerization appears to improve the activity of the gAd active site, as a C-terminally tethered version of ADP355 (termed ADP399, Table 1) exhibits a 10-fold decrease of K562 chronic myeloid leukemia cell proliferation rate compared to monomeric ADP355 (33), although this improvement is not obvious in animal models.

Another support for the identification of the correct active site comes from yet another screening of a virtual library against the three-dimensional structure of AdipoR1 (42). The best hit, a 7-mer peptide called Pep70 is strikingly similar to the minimal active site sequence (Table 1). Pep70 inhibits HSC-T6 cell proliferation with an $\mathrm{IC}_{50}$ of $5 \mu \mathrm{M}$, that is quite similar to ADP355 in this particular measure, casting some doubts to the validity of the biological assay selection.

\section{FIRST GENERATION OF SMALL MOLECULE ADIPONECTIN RECEPTOR AGONISTS}

Not much after the discovery of peptidomimetic ADP355, an extensive study used ADP355 as a reference compound to identify non-peptidic AdipoR agonists from a 10,000-member natural product library. Not surprisingly, even the best hit arctin retains only $3.5 \mu \mathrm{M}$ activity (43). Additional identified AdipoR1 agonists include arctigenin and gramine. En route to pulling out these compounds from the mixture, fluorescence polarization quantified the binding constants of ADP355 to AdipoR forms. Depending upon the location of the fluorescein label and the receptor subtype, the measured $K_{d}$ values range from $800 \mathrm{nM}$ to 1.7 $\mu \mathrm{M}$. In our experience, the presence of a bulky label decreases the affinity of peptide ligands to their binding partners by 50 to 100 -fold compared to naked peptides (44). Thus, these binding assays place the affinity of ADP355 to AdipoR somewhere at the low nM level, in agreement with the results of cellular adiponectin replacement experiments. It needs to be added that according to siRNA inhibition assays, ADP355 activates AdipoR1 stronger than it does AdipoR2 (29), a finding of some difference with the presented fluorescence polarization data.

Parallel to the initial hits, screening of another small molecule library identified an orally available AdipoR agonist called AdipoRon (45). AdipoRon is currently the most extensively studied non-peptidic adiponectin replacement therapy drug candidate.

\section{WIDE SPECTRUM OF IN VITRO AND IN VIVO ACTIVITIES OF ADIPORON}

AdipoRon (Figure 1) was selected from a chemical library at the Open Innovation Center for Drug Discovery in The University of Tokyo (no 108049) based on activation of AMPK and target validation with AdipoR siRNA inhibition in C2C12 myotubes (45). Another hit, no 112254 (Figure 2) has similar in vitro activity data, albeit structurally the two compounds are quite different except the p-cresol-piperidine fragment. The binding constants of AdipoRon to AdipoR1 and AdipoR2 are 1.8 and $3.1 \mu \mathrm{M}$. The $\mathrm{EC}_{50}$ value for AMPK activation is $\sim 10 \mu \mathrm{M}$ (45) in agreement with the receptor binding data. Similar $(5-10 \mu \mathrm{M})$ in vitro activities were observed in $\mathrm{C} 3 \mathrm{H} 10 \mathrm{~T} 1 / 2$ cells, where addition of AdipoRon increases phosphorylated AMPK and ACC levels, the latter being a downstream target of AMPK, and decreases the expression of adipogenic transcription factors $\mathrm{C} / \mathrm{EBP} \beta, \mathrm{C} / \mathrm{EBP} \alpha$, and PPAR $\gamma$ (46). Ensuing studies fully support AdipoRon as an adiponectin replacement therapy effector, although current pharmaceutical development go-nogo criteria are not in favor of pursuing a candidate with such a low cellular activity.

In the original report, orally administered AdipoRon at $50 \mathrm{mg} / \mathrm{kg}$ to genetically obese $\mathrm{db} / \mathrm{db}$ mice (lacking the leptin receptor) significantly reduces plasma glucose levels similarly in duration and extent to adiponectin protein injection. At the end of the 2-week treatment while the mice received normal diet, glucose intolerance, insulin resistance and dyslipidaemia are markedly ameliorated without affecting body weight, food intake, liver weight, and white adipose tissue weight. When the diet is switched to high fat content, AdipoRon rescues obesity-induced shortened lifespan of the $\mathrm{db} / \mathrm{db}$ mice. Very recently the efficacy of AdipoRon was studied in experimental periodontitis using diet-induced obese mice (47). In this model, the small molecule AdipoR agonist reduces osteoclast numbers and alveolar bone loss likely though inhibition of osteoclast differentiation.

AdipoRon is shown to elicit adiponectin-like activities in a wide spectrum of animal models. Covering all these reports are beyond the scope of this general review on adiponectin receptor response modifiers. Below is a brief selection focusing on the breadth of the studies. AdipoRon treatment of vascular smooth muscle cells (VSMC) leads to inhibition of platelet-derived growth factor-induced cell proliferation, DNA synthesis, and cyclin D1 expression with an $\mathrm{IC}_{50}$ of $25-50 \mu \mathrm{M}(48)$. In a mouse model of vascular remodeling caused by proliferation of VSMC, oral administration of $50 \mathrm{mg} / \mathrm{kg}$ AdipoRon diminishes arterial injury-induced neointima formation by $\sim 57 \%$. Likewise, orally administered in a mouse cardiac remodeling model, AdipoRon 
<smiles>O=C(COc1ccc(C(=O)c2ccccc2)cc1)NC1CCN(Cc2ccc(O)cc2)CC1</smiles>

AdipoRon<smiles>COc1ccc(CN2CCN(C(=O)CCNC(=O)c3ccc(C(C)(C)C)cc3O)CC2)cc1</smiles>

112254<smiles>O=C1Oc2cc(O)c(C3OCC(O)C(O)C(O)C3O)c(O)c2C(=O)C1c1ccc(O)c(O)c1</smiles>

GTDF

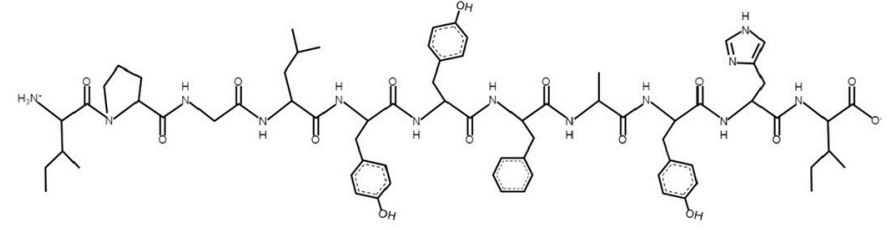

Globular adiponectin active site

FIGURE 2 | Chemical structures of the globular adiponectin active site and selected small molecule adiponectin receptor agonists.

alleviates cardiac hypertrophy and fibrosis induced by pressure overload and inhibits angiotensin II-induced TGF- $\beta 1$ expression as well as cardiac fibroblast differentiation through AMPK $\alpha$ activation (49).

The activity of AdipoRon was extensively studied in mouse models of diseases similar to those investigated for the efficacy of peptidic AdipoR agonists. AdipoRon inhibits pancreatic cancer cell proliferation, colony formation, and anchorage independent growth while increases apoptosis with an $\mathrm{IC}_{50}$ of $\sim 25 \mu \mathrm{M}$ (50). In an orthotopic pancreatic cancer model, AdipoRon at $5 \mathrm{mg} / \mathrm{kg}$ /day (administration mode not disclosed) for 14-days reduces tumor size and tumor area per tumor section by $50-75 \%$. Administered intraperitoneally at $1 \mathrm{mg} / \mathrm{kg} /$ day for 21-days to mice, AdipoRon reverses corticosterone-induced depression-like state (51). AdipoRon exerts pleiotropic actions on hippocampal neurogenesis, neuroinflammation, serotonergic neurotransmission, and the tryptophan metabolic pathway explaining its antidepressant properties. In mice with acute hepatic injury induced by d-galactosamine, AdipoRon attenuates liver inflammation as read by dwindling proinflammatory macrophage infiltration, shrinkage of pro-inflammatory cytokine production including TNF- $\alpha$, TGF- $\beta 1$, IL- $1 \beta$, and IL- 6 , meanwhile promoting AMPK activation by phosphorylation (52).

In a mouse model identical to that used for peptidomimetic ADP355, AdipoRon prevents the development of dermal fibrosis. At a concentration of $20 \mu \mathrm{M}$, AdipoRon blocks TGF$\beta$-induced fibrotic responses and given orally for 28 days significantly attenuates bleomycin-induced dermal thickness increase while augmenting the dermal white adipose tissue layer (53). When AdipoRon was directly compared with ADP355, low-density lipoprotein receptor expression was more extensive by using $25 \mathrm{nM}$ of the peptidomimetic than $10 \mu \mathrm{M}$ of the small molecule AdipoR agonist in HepG2 cells (37). A comparison of selected in vitro and in vivo efficacies of various adiponectin receptor response modifiers is found in Table 2.

\section{ADDITIONAL NON-PEPTIDIC ADIPONECTIN RECEPTOR AGONISTS}

Two additional flavonol-type compounds exhibit adiponectinlike activities. GTDF [6-C- $\beta$-d-glucopyranosyl-(2S,3S)- $(+)$ $5,7,3^{\prime}, 4^{\prime}$-tetrahydroxydihydroflavonol, Figure 2] is a natural analog of the dietary flavonoid quercetin (54). In a competition radioligand assay using $\mathrm{C} 2 \mathrm{C} 12$ myotubes, GTDF interacts with both AdipoRs, with a preference for AdipoR 1 at $10 \mathrm{nM}$ that is a magnitude better than the native compound quercetin and dihydroquercetin, the latter being the aglycon of GTDF. The AdipoR1 agonist induces adiponectin-associated signaling and enhances glucose uptake and fatty acid oxidation in vitro and improves metabolic health (elevation of glucose clearance, $\beta$-cell survival, reduced steatohepatitis, browned white adipose tissue, and improvement of lipid profile) in an AdipoR1-expressing but not an AdipoR1-depleted strain of diabetic mice (54). The dose of GTDF in this instance was $10 \mathrm{mg} / \mathrm{kg} /$ day orally for 30 days. Please note the remarkably improved pharmacological parameters of GTDF compared to AdipoRon.

Tiliroside is a glycosidic flavonoid and is found in several dietary plants (55). At a very high dose of $100 \mathrm{mg} / \mathrm{kg} /$ day, tiliroside enhances fatty acid oxidation through improved adiponectin signaling and ameliorates obesity-induced metabolic disorders including hyperinsulinemia and hyperlipidemia (56). 
TABLE 2 | Published activities of adiponectin receptor response modifiers.

\begin{tabular}{|c|c|c|c|}
\hline Molecule & $\begin{array}{l}\text { in vitro activity } \\
\text { approx. } \mathrm{IC}_{50}\end{array}$ & $\begin{array}{l}\text { in vivo efficacy } \\
\text { ( } \mathrm{mg} / \mathrm{kg} \text {, duration, administration mode) }\end{array}$ & References \\
\hline AdipoRon & $\begin{array}{l}\text { Activation of LDLR } \\
5 \mu \mathrm{M}\end{array}$ & $\begin{array}{l}\text { Reversal of corticosterone-induced depression } \\
(1 \mathrm{mg} / \mathrm{kg} \text { in mice, ip, } 21 \mathrm{~d})\end{array}$ & $\begin{array}{l}(37) \\
(51)\end{array}$ \\
\hline GTDF & $\begin{array}{l}\text { Enhanced of glucose uptake } \\
\text { Activity observed at } 10 \mathrm{nM}\end{array}$ & $\begin{array}{l}\text { Improvement of metabolic health } \\
10 \mathrm{mg} / \mathrm{kg}, \mathrm{po}, 30 \mathrm{~d}\end{array}$ & (54) \\
\hline ADP355 & $\begin{array}{l}\text { Activation of LDLR } \\
25 \mathrm{nM}\end{array}$ & $\begin{array}{l}\text { Complete prevention of protease-inhibitor-induced } \\
\text { memory decline } \\
(1 \mathrm{mg} / \mathrm{kg} \text { in mice, ip, } 14 \mathrm{~d})\end{array}$ & $\begin{array}{l}(37) \\
(38)\end{array}$ \\
\hline ADP399 & $\begin{array}{l}\text { Cancer cell proliferation inhibition } \\
10 \mathrm{nM}\end{array}$ & & (33) \\
\hline Pep70 & $\begin{array}{l}\text { HSC proliferation inhibition } \\
10 \mu \mathrm{M}\end{array}$ & & $(42)$ \\
\hline BHD-1028 & $\begin{array}{l}\text { AMPK activation } \\
<800 \mathrm{nM}\end{array}$ & & $(41)$ \\
\hline ADP400 & $900\left(E_{50}\right)$ & & (33) \\
\hline
\end{tabular}

LDLR, low density lipoprotein receptor; ip, intraperitoneally; po, orally; HSC, hepatic stellate cells; AMPK, $5^{\prime}$ adenosine monophosphate-activated protein kinase.

Nevertheless, tiliroside treatment does not suppress body weight gain and visceral fat accumulation in obese mice. While AdipoR1 and AdipoR2 mRNA expression levels are up-regulated by tiliroside treatment, direct binding to the receptors have not been reported yet.

\section{ANTAGONISTS}

Currently only a very limited number of AdipoR antagonists exist although these could find application in diseases characterized by adiponectin overproduction. In this regard, plasma adiponectin levels are reproducibly higher in severe rheumatoid arthritis patients than in the control group (57). Likewise, both AdipoR subtypes are overexpressed in lesional compared to nonlesional areas of osteoarthritis cartilage (58). Conceivably AdipoR antagonists may find applicability as a therapy option in arthritic diseases. Nevertheless, this statement has to be taken with a grain of salt, as tocilizumab (an IL-6 receptor antagonist) treatment of rheumatoid arthritis patients results in both improved clinical findings and increased serum adiponectin levels (59). Another potential initiative for finding adiponectin receptor antagonists is that both AdipoR1 and AdipoR2 are involved in energy metabolism but were reported to have opposing effects suggesting a potential therapeutic utility of negative AdipoR response modifiers in some cardiovascular conditions (60). Indeed, high adiponectin concentrations are associated with increased cardiovascular mortality in patients with congestive heart failure $(61,62)$, in coronary heart disease and myocardial infarction $(63,64)$ as well as in chronic kidney disease (65). These studies suggest that once a chronic condition is established, the reverse to traditional adiponectin epidemiology can be observed (66).

However, given the multiple physiological advantages of high levels or circulating adiponectin, the clinical development of negative adiponectin receptor response modifiers without noticeable negative side effects seems extremely difficult at this point. Yet, AdipoR antagonists would represent a much-desired target validation tool for AdipoR agonist drug development.

The first AdipoR antagonist was derived by the agonist peptidomimetic ADP355 by using peptide chemistry tools of the trade proven successful in converting leptin-based ObR agonist peptides into picomolar antagonists (67). Screening of active site library but this time focusing on antagonist properties, a novel octapeptide (ADP400, Table 1) was designed that counteracts ADP355- and its dimer-mediated effects on cancer cell growth at nanomolar concentrations [(33); Table 2]. ADP400 induces mitogenic effects in MCF-7 breast cancer cells perhaps due to antagonizing endogenous adiponectin actions or acting as an inverse agonist.

A ceramidase inhibitor, 1S,2R-D-erythro-2-Nmyristoylamino-1-phenyl-1-propanol (MAPP), antagonizes AdipoR signaling at low nanomolar concentrations likely through inverse agonist properties (68). The same yeast-based assay identified TNF $\alpha$ as a potential AdipoR1 antagonist albeit at a 10 -fold reduced activity level compared to MAPP. While in biochemistry terms the findings are noteworthy, the fact that agonist is not required to activate the proxy downstream signaling pathway casts shadow to potential significance of the findings in drug development pharmacology.

\section{ADIPONECTIN ACTION MIMICKING PEPTIDES AND PROTEINS}

Biooligomers and biopolymers can either induce adiponectin production in cells and tissues or their synthesis can be triggered by adiponectin in a downstream process. Since these peptides and proteins may serve as adiponectin replacement effectors in various biological processes their potential as therapeutic agents are briefly discussed here.

Upon exposure to adiponectin, B-cells are able to inhibit Tcell trafficking by secreting a 14-mer peptide with a sequence SVTEQGAELSNEER and named PEPITEM (69). PEPITEM 
is a fragment of the 14.3.3. $\zeta \delta$ protein and binds cadherin15 on endothelial cells, promoting synthesis and release of sphingosine-1 phosphate ultimately inhibiting T-cell trafficking. In various mouse models of hepatic ischemia-reperfusion injury, Salmonella typhimurium infection, peritonitis, uveitis and Sjögren's syndrome, PEPITEM reduces T-cell recruitment into inflamed tissues. From a drug development point of view, the presented animal models employ very high peptide doses (15 $\mathrm{mg} / \mathrm{kg}$ bolus and $5 \mathrm{mg} / \mathrm{kg}$ chronic treatments intraperitoneally or $6 \mu \mathrm{g}$ peptide directly to the eye). These are significantly higher than those reported for ADP355, an adiponectin-derived anti-inflammatory therapy option (vide infra).

Secretion of adiponectin is a highly complex process that is influenced by many factors (70). A long range of agents can increase adiponectin production in cells and tissues, but currently these are far from being therapeutically significant. The following is just as a brief outline. The TNF$\alpha$ antagonist etanercept increases AMPK phosphorylation in mice, and exerts cardioprotective effects partially due to the upregulation of adiponectin (71). Likewise, safflomide (Ncaffeoyltryptamine), a serotonin receptor antagonist, upregulates adiponectin expression in high fat diet-fed rats, resulting in significant reduction in body weight, visceral fat, and improved insulin resistance (72). Improved hepatic insulin sensitivity by the cannabinoid receptor 1 antagonist rimonabant is due to upregulation of adiponectin (73). Rimonabant in high fat-fed dogs enhances hepatic insulin clearance, a process that appears to be linked to an upregulation of the adiponectin pathway.

Activation of PPAR- $\gamma$, one of the central regulators of adipocyte biology, improves the secretory profile of the adipose tissue, promoting the release of insulin-sensitizing adipokines, including adiponectin, and reducing inflammatory cytokines (74). It is not surprising that upregulation of adiponectin activity is frequently attempted by enhancing PPAR- $\gamma$-mediated biological processes. Rosiglitazone (trade name Avandia) is a small molecule antidiabetic drug and is a PPAR- $\gamma$ agonist. A recent mode-of-action study revealed that rosiglitazone, similar to other thiazolidinedione insulin sensitizers, elicits an adiponectin-mediated action at the adipose tissue-liver axis in obese rats (75). In this instance, rosiglitazone ameliorates hepatic and systemic insulin resistance, hepatic inflammation, and fatty liver. Rosiglitazone passes the blood-brain barrierand elicits antidepressant- and anxiolytic-like behavioral effects in mice accompanied by a concurrent increase in serum adiponectin levels (76). Pretreatment with the PPAR- $\gamma$-selective antagonist GW9662 blocks rosiglitazone-induced adiponectin production and the central nervous system-resident effects validating PPAR- $\gamma$ as target of the drug and its positive regulatory role in adiponectin expression. CCK-8 (commercial name Sincalide) is the C-terminal octapeptide fragment of cholecystokinin. CCK-8 stimulates adiponectin production in rat white adipose tissue through a mechanism involving both AKT and PPAR- $\gamma$ (77). Since CCK- 8 actions are only observed in the presence of insulin, it is contemplated to have translational value in novel insulin-sensitizing therapies. Another target validation assay used telmisartan, a dual angiotensin II receptor antagonist and partial PPAR- $\gamma$ receptor agonist to document a significant reduction of myocardial damage induced by ischemia/reperfusion where the activity is associated with increased adiponectin production and a reduction in certain markers of inflammation (78).

The previous report leads us to additional PPAR subtypes. Fimasartan is a non-peptide angiotensin II receptor antagonist used for the treatment of hypertension and heart failure. In HepG2 cells and liver tissues, fimasartan increases the protein levels of PPAR- $\delta$. It also increases the adiponectin level in visceral fat tissues (79). In target validation experiments, the anti-adipogenic effects can be offset with the PPAR- $\delta$ antagonist GSK0660. In differentiated adipocytes the PPAR- $\beta / \delta$ agonist GW501516 induces adipocyte differentiation and the expression of adiponectin in a concentration-dependent manner and ultimately improves insulin sensitivity by increasing the expression of the insulin receptor (80). In an only partially elucidated yet nutritionally interesting aspect, dietary capsaicin may reduce obesity-induced glucose intolerance by suppression of inflammatory responses and enhancement of fatty acid oxidation in adipose tissue and the liver (81). During this process, inflammatory cytokine production decreases and adiponectin in the adipose tissue and PPAR- $\alpha$ in the liver increases.

While some of these studies did experiment with concentration- and dose-response characterization of existing drugs and drug-like adiponectin activity enhancers, their full pharmacological characterization relative to direct adiponectin replacement therapies seems to be available only in a distant future.

\section{CONCLUSIONS AND FURTHER PERSPECTIVE}

Based on the dire need for adiponectin receptor response modifiers and the numerous promising candidates in preclinical development it is very likely that adiponectin receptor agonists will enter human clinical trials in the near future. Allysta Pharmaceuticals plans to initiate human trials in late 2019 against dry eye disease using ADP355 as eye drops. From the small molecule agonists listed here, at the time of writing this report, AdipoRon is not subject to any human trial (82). A potential reason for this is that systemic drug formulations have to go through stricter toxicity measures than topical dosage forms.

Drugs targeting adipokine receptors frequently exhibit bell shaped activity curves in vitro and a dose-dependent antagonistagonist switch in vivo, leptin variations being prime examples (44). With the numerous proven activities of adiponectin receptor agonists and possibly antagonists we are facing a long process of identifying optimal doses, dose timing and administration modes or formulations that induce only the desired human responses without any unwanted side effects. In addition, coming again from the leptin experience, elevated levels of adipokines may not necessarily mean high levels of active circulating proteins. For example, elevated adiponectin levels may indicate disease progression where adiponectin plays a protective role and is excessively produced. Strong evidence for a possible correlation between adiponectin levels and progressive 
liver fibrosis in patients with chronic hepatitis B virus infection is observed (83).

In this regard, the generally observable fast renal clearance of peptide drugs (84) may even come handy. Peptides hit their receptors fast, keep them activated/deactivated long but leave the circulation within $1 \mathrm{~h}$. With proper dosing, formulation and perhaps fine-tuning the sequences, peptidic adipokine receptor

\section{REFERENCES}

1. Barb D, Williams CJ, Neuwirth AK, Mantzoros CS. Adiponectin in relation to malignancies: A review of existing basic research and clinical evidence. $\mathrm{Am} J$ Clin Nutr. (2007) 86:s858-66. doi: 10.1093/ajcn/86.3.858S

2. Schaffler A, Scholmerich J, Buechler C. Mechanisms of disease: adipokines and breast cancer - endocrine and paracrine mechanisms that connect adiposity and breast cancer. Nat Clin Pract Endocrinol Metab. (2007) 3:345-54. doi: $10.1038 /$ ncpendmet 0456

3. Ziemke F, Mantzoros CS. Adiponectin in insulin resistance: Lessons from translational research. Am J Clin Nutr. (2010) 91:258S-61. doi: 10.3945/ajcn.2009.28449C

4. Richards AA, Stephens T, Charlton HK, Jones A, Macdonald GA, Prins $\mathrm{JB}$, et al. Adiponectin multimerization is dependent on conserved lysines in the collagenous domain: evidence for regulation of multimerization by alterations in posttranslational modifications. Mol Endocrinol. (2006) 20:1673-87. doi: 10.1210/me.2005-0390

5. Fang X, Sweeney G. Mechanisms regulating energy metabolism by adiponectin in obesity and diabetes. Biochem Soc Trans. (2006) 34:798-801. doi: 10.1042/BST0340798

6. Yamauchi T, Kamon J, Ito Y, Tsuchida A, Yokomizo T, Kita S, et al. Cloning of adiponectin receptors that mediate antidiabetic metabolic effects. Nature. (2003) 423:762-9. doi: 10.1038/nature01705

7. Kadowaki T, Yamauchi T. Adiponectin receptor signaling: A new layer to the current model. Cell Metab. (2011) 13:123-4. doi: 10.1016/j.cmet.2011.01.012

8. Brochu-Gaudreau K, Rehfeldt C, Blouin R, Bordignon V, Murphy BD, Palin MF. Adiponectin action from head to toe. Endocrine. (2010) 37:11-32. doi: 10.1007/s12020-009-9278-8

9. Lim S, Quon MJ, Koh KK. Modulation of adiponectin as a potential therapeutic strategy. Atherosclerosis. (2014) 233:721-8. doi: 10.1016/j.atherosclerosis.2014.01.051

10. Achari AF, Jain SK. Adiponectin, a therapeutic target for obesity, diabetes, and endothelial dysfunction. Int J Mol Sci. (2017) 18:E1321. doi: 10.3390/ijms18061321

11. Monks M, Irakleidis F, Tan PH. Complex interaction of adiponectin-mediated pathways on cancer treatment: a novel therapeutic target. J Cancer Metastasis Treat. (2019) 5:24. doi: 10.20517/2394-4722.2018.79

12. Jin $\mathrm{Z}, \mathrm{Du} \mathrm{Y}$, Schwaid AG, Asterholm IW, Scherer PE, Saghatelian A, et al. Maternal adiponectin controls milk composition to prevent neonatal inflammation. Endocrinology. (2015) 156:1504-13. doi: 10.1210/en.2014-1738

13. Udomsinprasert $\mathrm{W}$, Honsawek $\mathrm{S}$, Poovorawan $\mathrm{Y}$. Adiponectin as a novel biomarker for liver fibrosis. World J Hepatol. (2018) 10:708-18. doi: 10.4254/wjh.v10.i10.708

14. Bloemer J, Pinky PD, Govindarajulu M, Hong H, Judd R, Amin RH, et al. Role of adiponectin in central nervous system disorders. Neural Plast. (2018) 2018:4593530. doi: 10.1155/2018/4593530

15. Waki H, Yamauchi T, Kamon J, Ito Y, Uchida S, Kita S, et al. Impaired multimerization of human adiponectin mutants associated with diabetes. Molecular structure and multimer formation of adiponectin. $J$ Biol Chem. (2003) 278:40352-63. doi: 10.1074/jbc.M300365200

16. Fisher FM, Trujillo ME, Hanif W, Barnett AH, McTernan PG, Scherer PE, et al. Serum high molecular weight complex of adiponectin correlates better with glucose tolerance than total serum adiponectin in Indo-Asian males. Diabetologia. (2005) 48:1084-87. doi: 10.1007/s00125-005-1758-7

17. Heiker JT, Klöting N, Blüher M, Beck-Sickinger AG. Access to gram scale amounts of functional globular adiponectin from E. coli inclusion bodies by modifiers can be tailored to induce only desired activities without expanding the pharmacology for an extended period of time and potentially bringing out toxic reactions in patients.

\section{AUTHOR CONTRIBUTIONS}

LO collected the cited papers and wrote the entire manuscript. alkaline-shock solubilization. Biochem Biophys Res Commun. (2010) 398:32-7. doi: 10.1016/j.bbrc.2010.06.020

18. Wang Y, Xu A, Knight C, Xu LY, Cooper GJ. Hydroxylation and glycosylation of the four conserved lysine residues in the collagenous domain of adiponectin. Potential role in the modulation of its insulin-sensitizing activity. J Biol Chem. (2002) 277:19521-9. doi: 10.1074/jbc.M200601200

19. Wang ZV, Scherer PE. Adiponectin, the past two decades. J Mol Cell Biol. (2016) 8:93-100. doi: 10.1093/jmcb/mjw011

20. Wijesekara N, Krishnamurthy M, Bhattacharjee A, Suhail A, Sweeney G, Wheeler MB. Adiponectin-induced ERK and Akt phosphorylation protects against pancreatic $\beta$-cell apoptosis and increases insulin gene expression and secretion. J Biol Chem. (2010) 285:33623-31. doi: 10.1074/jbc.M109.085084

21. Dadson K, Liu Y, Sweeney G. Adiponectin action: a combination of endocrine and autocrine/paracrine effects. Front Endocrinol. (2011) 2:62. doi: $10.3389 /$ fendo.2011.00062

22. Zhang L, Wen K, Han X, Liu R, Qu Q. Adiponectin mediates antiproliferative and apoptotic responses in endometrial carcinoma by the AdipoRs/AMPK pathway. Gynecol Oncol. (2015) 137:311-20. doi: 10.1016/j.ygyno.2015.02.012

23. Wannamethee SG, Welsh P, Whincup PH, Sawar N, Thomas MC, Gudnarsson $\mathrm{V}$, et al. High adiponectin and increased risk of cardiovascular disease and mortality in asymptomatic older men: Does NT-proBNP help to explain this association? Eur J Cardiovasc Prev Rehabil. (2011) 18:65-71. doi: 10.1097/HJR.0b013e32833b09d9

24. Mao X, Kikani CK, Riojas RA, Langlais P, Wang L, Ramos FJ, et al. APPL1 binds to adiponectin receptors and mediates adiponectin signalling and function. Nat Cell Biol. (2006) 8:516-23. doi: 10.1038/ncb1404

25. Potter JJ, Mezey E. Acetaldehyde increases endogenous adiponectin and fibrogenesis in hepatic stellate cells but exogenous adiponectin inhibits fibrogenesis. Alcohol Clin Exp Res. (2007) 31:2092-100. doi: 10.1111/j.1530-0277.2007.00529.x

26. Miller RA, Chu Q, Le Lay J, Scherer PE, Ahima RS, Kaestner KH, et al. Adiponectin suppresses gluconeogenic gene expression in mouse hepatocytes independent of LKB1-AMPK signaling. J Clin Invest. (2011) 212:2518-28. doi: $10.1172 /$ JCI 45942

27. Fuerst M, Taylor CG, Wright B, Tworek L, Zahradka P. Inhibition of smooth muscle cell proliferation by adiponectin requires proteolytic conversion to its globular form. J Endocrinol. (2012) 215:107-17. doi: 10.1530/JOE-12-0021

28. Dry Eye Zone (2019). Available online at: https://www.dryeyezone.com/dryeye-drug-pipeline (accessed July 31, 2019).

29. Otvos L Jr., Haspinger E, La Russa F, Maspero F, Graziano P, Kovalszky I, et al. Design and development of a peptide-based adiponectin receptor agonist for cancer treatment. BMC Biotechnol. (2011) 11:90. doi: 10.1186/1472-6750-11-90

30. Tomas E, Tsao TS, Saha AK, Murrey HE, Zhang CC, Itani SI, et al. Enhanced muscle fat oxidation and glucose transport by ACRP30 globular domain: Acetyl-CoA carboxylase inhibition and AMP-activated protein kinase activation. Proc Natl Acad Sci USA. (2002) 99:16309-13. doi: $10.1073 /$ pnas.222657499

31. Wu X, Motoshima H, Mahadev K, Stalker TJ, Scalia R, Goldstein BJ Involvement of AMP-activated protein kinase in glucose uptake stimulated by the globular domain of adiponectin in primary rat adipocytes. Diabetes. (2003) 52:1355-63. doi: 10.2337/diabetes.52.6.1355

32. Sayeed M, Gautam S, Verma DP, Afshan T, Kumari T, Srivastava AK, et al A collagen domain-derived short adiponectin peptide activates APPL1 and AMPK signaling pathways and improves glucose and fatty acid metabolisms. J Biol Chem. (2018) 293:13509-23. doi: 10.1074/jbc.RA118.001801 
33. Otvos L Jr., Knappe D, Hoffmann R, Kovalszky I, Olah J, Hewitson TD, et al. Development of second generation peptides modulating cellular adiponectin receptor responses. Front Chem. (2014) 2:93. doi: 10.3389/fchem.2014.0 0093

34. Marangoni RG, Masui Y, Fang F, Korman B, Lord G, Lee J, et al. Adiponectin is an endogenous anti-fibrotic mediator and therapeutic target. Sci Rep. (2017) 7:4397. doi: 10.1038/s41598-017-04162-1

35. Kumar P, Smith T, Rahman K, Mells JE, Thorn NE, Saxena NK, et al. Adiponectin modulates focal adhesion disassembly in activated hepatic stellate cells: implication for reversing hepatic fibrosis. FASEB J. (2014) 28:5172-83. doi: 10.1096/fj.14-253229

36. Kumar P, Smith T, Rahman K, Thorn NE, Anania FA. Adiponectin agonist ADP355 attenuates $\mathrm{CCl}_{4}$-induced liver fibrosis in mice. PLoS ONE. (2014) 9:e110405. doi: 10.1371/journal.pone.0110405

37. Sun L, Yang X, Li Q, Zeng P, Liu Y, Liu L, et al. Activation of adiponectin receptor regulates proprotein convertase subtilisin/kexin type 9 expression and inhibits lesions in ApoE-deficient mice. Arterioscler Thromb Vasc Biol. (2017) 37:1290-300. doi: 10.1161/ATVBAHA.117.309630

38. Pepping JK, Otvos L Jr., Surmacz E, Gupta S, Keller JN, Bruce-Keller AJ. Designer adiponectin receptor agonist stabilizes metabolic function and prevents brain injury caused by HIV protease inhibitors. J Neuroimmune Pharmacol. (2014) 9:388-98. doi: 10.1007/s11481-014-9529-1

39. Wang H, Zhang H, Zhang Z, Huang B, Cheng X, Wang D, et al. Adiponectinderived active peptide ADP355 exerts anti-inflammatory and anti-fibrotic activities in thioacetamide-induced liver injury. Sci Rep. (2016) 6:19445. doi: 10.1038/srep19445

40. Otvos L Jr., Kovalszky I, Olah J, Coroniti R, Knappe D, Nollmann FI, et al. Optimization of adiponectin-derived peptides for inhibition of cancer cell growth and signaling. Biopolymers. (2015) 104:156-66. doi: 10.1002/bip. 22627

41. Kim S, Lee Y, Kim JW, Son YJ, Ma MJ, Um JH, et al. Discovery of a novel potent peptide agonist to adiponectin receptor 1. PLOS ONE. (2018) 13:e0199256. doi: 10.1371/journal.pone.0199256

42. Ma L, Zhang Z, Xue X, Wan Y, Ye B, Lin K. A potent peptide as adiponectin receptor 1 agonist to against fibrosis. J Enzyme Inhib Med Chem. (2017) 32:624-31. doi: 10.1080/14756366.2017.1284067

43. Sun Y, Zang Z, Zhong L, Wu M, Su Q, Gao X, et al. Identification of adiponectin receptor agonist utilizing a fluorescence polarization based high throughput assay. PLoS ONE. (2013) 8:e63354. doi: 10.1371/journal.pone.0063354

44. Otvos L Jr., Vetter SW, Koladia M, Knappe D, Schmidt R, Ostorhazi E, et al. The designer leptin antagonist peptide Allo-aca compensates for short serum half-life with very tight binding to the receptor. Amino Acids. (2014) 46:873-82. doi: 10.1007/s00726-013-1650-6

45. Okada-Iwabu M, Yamauchi T, Iwabu M, Honma T, Hamagami KI, Matsuda K, et al. A small-molecule AdipoR agonist for type 2 diabetes and short life in obesity. Nature. (2013) 503:493-9. doi: 10.1038/nature 12656

46. Wang SJ, Lu WY, Liu KY. Adiponectin receptor agonist AdipoRon suppresses adipogenesis in C3H10T1/2 cells through the adenosine monophosphateactivated protein kinase signaling pathway. Mol Med Rep. (2017) 16:7163-9. doi: $10.3892 / \mathrm{mmr} .2017 .7450$

47. Wu X, Qiu W, Hu Z, Lian J, Liu Y, Zhu X, et al. An adiponectin receptor agonist reduces type 2 diabetic periodontitis. J Dent Res. (2019) 98:313-21. doi: $10.1177 / 0022034518818449$

48. Fairaq A, Shawky NM, Osman I, Pichavaram P, Segar L. AdipoRon, an adiponectin receptor agonist, attenuates PDGF-induced VSMC proliferation through inhibition of mTOR signaling independent of AMPK: Implications toward suppression of neointimal hyperplasia. Pharmacol Res. (2017) 119:289-302. doi: 10.1016/j.phrs.2017.02.016

49. Zhang N, Wei WY, Liao HH, Yang Z, Hu C, Wang SS, et al. AdipoRon, an adiponectin receptor agonist, attenuates cardiac remodeling induced by pressure overload. J Mol Med. (2018) 96:1345-57. doi: 10.1007/s00109-018-1696-8

50. Messaggio F, Mendonsa AM, Castellanos J, Nagathihalli NS, Gorden L, Merchant NB, et al. Adiponectin receptor agonists inhibit leptin induced pSTAT3 and in vivo pancreatic tumor growth. Oncotarget. (2017) 8:85378-91. doi: 10.18632 /oncotarget.19905
51. Nicolas S, Debayle D, Béchade C, Maroteaux L, Gay AS, Bayer P, et al. AdipoRon, an adiponectin receptor agonist acts as an antidepressant and metabolic regulator in a mouse model of depression. Transl Psychiatry. (2018) 8:159. doi: 10.1038/s41398-018-0210-y

52. Wang Y, Wan Y, Ye G, Wang P, Xue X, Wu G, et al. Hepatoprotective effects of AdipoRon against d-galactosamine-induced liver injury in mice. Eur J Pharm Sci. (2016) 93:123-31. doi: 10.1016/j.ejps.2016.08.017

53. Yamashita $\mathrm{T}$, Lakota $\mathrm{K}$, Taniguchi $\mathrm{T}$, Yoshizaki A, Sato $\mathrm{S}$, Hong $\mathrm{W}$, et al. An orally-active adiponectin receptor agonist mitigates cutaneous fibrosis, inflammation and microvascular pathology in a murine model of systemic sclerosis. Sci Rep. (2018) 8:11843. doi: 10.1038/s41598-018-29901-w

54. Singh AK, Joharapurkar AA, Khan MP, Mishra JS, Singh N, Yadav M, et al. Orally active osteoanabolic agent GTDF binds to adiponectin receptors, with a preference for AdipoR1, induces adiponectin-associated signaling, and improves metabolic health in a rodent model of diabetes. Diabetes. (2014) 63:3530-44. doi: 10.2337/db13-1619

55. Luhata LP, Luhata WG. Tiliroside: biosynthesis, bioactivity and structure activity relationship (SAR) - A review. J Phytophatmacol. (2017) 6:343-8.

56. Goto T, Teraminami A, Lee JY, Ohyama K, Funakoshi K, Kim YI, et al. Tiliroside, a glycosidic flavonoid, ameliorates obesity-induced metabolic disorders via activation of adiponectin signaling followed by enhancement of fatty acid oxidation in liver and skeletal muscle in obese-diabetic mice. J Nutr Biochem. (2012) 23:768-76. doi: 10.1016/j.jnutbio.2011.04.001

57. Ebina K, Fukuhara A, Ando W, Hirao M, Koga T, Oshima K, et al. Serum adiponectin concentrations correlate with severity of rheumatoid arthritis evaluated by extent of joint destruction. Clin Rheumatol. (2009) 28:445-51. doi: 10.1007/s10067-008-1074-y

58. Kang EH, Lee YJ, Kim TK, Chang CB, Chung JH, Shin K, et al. Adiponectin is a potential catabolic mediator in osteoarthritis cartilage. Arthritis Res Ther. (2010) 12:R231. doi: 10.1186/ar3218

59. Fioravanti A, Tenti S, Bacarelli MR, Damiani A, Li Gobbi F, Bandinelli F, et al. Tocilizumab modulates serum levels of adiponectin and chemerin in patients with rheumatoid arthritis: Potential cardiovascular protective role of IL-6 inhibition. Clin Exp Rheumatol. (2019) 37:293-300.

60. Bjursell M, Ahnmark A, Bohlooly YM, William-Olsson L, Rhedin M, Peng XR, et al. Opposing effects of adiponectin receptors 1 and 2 on energy metabolism. Diabetes. (2007) 56:583-93. doi: 10.2337/db06-1432

61. Kistorp C, Faber J, Galatius S, Gustafsson F, Frystyk J, Flyvbjerg A, et al. Plasma adiponectin, body mass index, and mortality in patients with chronic heart failure. Circulation. (2005) 112:1756-62. doi: 10.1161/CIRCULATIONAHA.104.530972

62. George J, Patal S, Wexler D, Sharabi Y, Peleg E, Kamari Y, et al. Circulating adiponectin concentrations in patients with congestive heart failure. Heart. (2006) 92:1420-4. doi: 10.1136/hrt.2005.083345

63. Cavusoglu E, Ruwende C, Chopra V, Yanamadala S, Eng C, Clark LT, et al. Adiponectin is an independent predictor of all-cause mortality, cardiac mortality, and myocardial infarction in patients presenting with chest pain. Eur Heart J. (2006) 27:2300-9. doi: 10.1093/eurheartj/ehl153

64. Pilz S, Mangge H, Wellnitz B, Seelhorst U, Winkelmann BR, Tiran B, et al. Adiponectin and mortality in patients undergoing coronary angiography. $J$ Clin Endocrinol Metab. (2006) 91:4277-86. doi: 10.1210/jc.2006-0836

65. Menon V, Li L, Wang X, Green T, Balakrishnan V, Madero M, et al. Adiponectin and mortality in patients with chronic kidney disease. J Am Soc Nephrol. (2006) 17:2599-606. doi: 10.1681/ASN.2006040331

66. Rathmann W, Herder C. Adiponectin and cardiovascular mortality: evidence for "reverse epidemiology". Hormone Metabolic Res. (2007) 39:1-2. doi: 10.1055/s-2007-958630

67. Otvos L Jr., Surmacz E. Targeting the leptin receptor: a potential new mode of treatment for breast cancer. Expert Rev Anticancer Ther. (2011) 11:1147-50. doi: 10.1586/era.11.109

68. Kupchak BR, Garitaonandia I, Villa NY, Smith JL, Lyons TJ. Antagonism of human adiponectin receptors and their membrane progesterone receptor paralogs by TNF $\alpha$ and a ceramidase inhibitor. Biochemistry. (2009) 48:5504-6. doi: 10.1021/bi9006258

69. Chimen M, McGettrick HM, Apta B, Kuravi SJ, Yates CM, Kennedy A, et al. Homeostatic regulation of $\mathrm{T}$ cell trafficking by a B cell-derived peptide is impaired in autoimmune and chronic inflammatory disease. Nat Med. (2015) 21:467-75. doi: $10.1038 / \mathrm{nm} .3842$ 
70. Wang ZV, Schraw TD, Kim JY, Khan T, Rajala MW, Follenzi A, et al. Secretion of the adipocyte-specific secretory protein adiponectin critically depends on thiol-mediated protein retention. Mol Cell Biol. (2007) 27:3716-31. doi: 10.1128/MCB.00931-06

71. Gao C, Liu Y, Yu Q, Yang Q, Li B, Sun L, et al. TNF- $\alpha$ antagonism ameliorates myocardial ischemia-reperfusion injury in mice by upregulating adiponectin. Am J Physiol Heart Circ Physiol. (2015) 308:H1583-91. doi: 10.1152/ajpheart.00346.2014

72. Park JB, Wang TT. Safflomide increases the expression of adiponectin in vitro and in vivo: Potential implication for hypoadiponectemia, visceral obesity, and insulin resistance. J Agric Food Chem. (2012) 60:4048-52. doi: 10.1021/jf2044108

73. Kabir M, Iyer MS, Richey JM, Woolcott OO, Asare Bediako I, Wu Q, et al. $\mathrm{CB} 1 \mathrm{R}$ antagonist increases hepatic insulin clearance in fat-fed dogs likely via upregulation of liver adiponectin receptors. Am J Physiol Endocrinol Metab. (2015) 309:E747-758. doi: 10.1152/ajpendo.00196.2015

74. Astapova O, Leff T. Adiponectin and PPAR $\gamma$ : cooperative and interdependent actions of two key regulators of metabolism. Vitam Horm. (2012) 90:143-62. doi: 10.1016/B978-0-12-398313-8.00006-3

75. Li J, Xue YM, Zhu B, Pan YH, Zhang Y, Wang C, et al. Rosiglitazone elicits an adiponectin-mediated insulin-sensitizing action at the adipose tissue-liver axis in Otsuka Long-Evans Tokushima fatty rats. J. Diabetes Res. (2018) 2018:4627842. doi: 10.1155/2018/4627842

76. Guo M, Li C, Lei Y, Xu S, Zhao D, Lu XY. Role of the adipose PPAR $\gamma$ adiponectin axis in susceptibility to stress and depression/anxiety-related behaviors. Mol Psychiatry. (2017) 22:1056-68. doi: 10.1038/mp.2016.225

77. Plaza A, Merino B, Del Olmo N, Ruiz-Gayo M. A cholecystokinin receptor agonist, (CCK-8), induces adiponectin production in rat white adipose tissue. Br J Pharmacol. (2019) 176:2678-90. doi: 10.1111/bph.14690

78. Rinaldi B, Di Filippo C, Capuano A, Donniacuo M, Sodano L, Ferraraccio F, et al. Adiponectin elevation by telmisartan ameliorates ischaemic myocardium in Zucker diabetic fatty rats with metabolic syndrome. Diabetes Obes Metab. (2012) 14:320-8. doi: 10.1111/j.1463-1326.2011.01527.x
79. Lee YJ, Jang YN, Han YM, Kim HM, Jeong JM, Seo HS. Fimasartan ameliorates nonalcoholic fatty liver disease through PPAR $\delta$ regulation in hyperlipidemic and hypertensive conditions. PPAR Res. (2017) 2017:8048720. doi: $10.1155 / 2017 / 8048720$

80. Kim WJ, Lee W, Jung Y, Jang HJ, Kim YK, Kim SN. PPAR $\beta / \delta$ agonist GW501516 inhibits TNF $\alpha$-induced repression of adiponectin and insulin receptor in 3T3-L1 adipocytes. Biochem Biophys Res Commun. (2019) 510:621-8. doi: 10.1016/j.bbrc.2019.02.013

81. Kang JH, Goto T, Han IS, Kawada T, Kim YM, Yu R. Dietary capsaicin reduces obesity-induced insulin resistance and hepatic steatosis in obese mice fed a high-fat diet. Obesity. (2010) 18:780-7. doi: 10.1038/oby.2009.301

82. US National Library of Medicine (2019). Available online at: https:// clinicaltrials.gov (accessed July 31, 2019).

83. Hsu CS, Liu WL, Chao YC, Lin HH, Tseng TC, Wang CC, et al. Adipocytokines and liver fibrosis stages in patients with chronic hepatitis B virus infection. Hepatol Int. (2015) 9:231-42. doi: 10.1007/s12072-015-9616-2

84. Otvos L Jr., Wade JD. Current challenges in peptide-based drug discovery. Front Chem. (2014) 2:62. doi: 10.3389/fchem.2014.00062

Conflict of Interest Statement: LO is President of OLPE, LLC, a pharmaceutical consulting firm and co-inventor of the issued US patent on ADP355. LO is a senior consultant of Allysta, Inc., a biotechnology company focusing on the clinical development of ADP355 against various human diseases.

The reviewer GS declared a shared affiliation, though no other collaboration, with the author to the handling editor.

Copyright (C) 2019 Otvos. This is an open-access article distributed under the terms of the Creative Commons Attribution License (CC BY). The use, distribution or reproduction in other forums is permitted, provided the original author(s) and the copyright owner(s) are credited and that the original publication in this journal is cited, in accordance with accepted academic practice. No use, distribution or reproduction is permitted which does not comply with these terms. 